\title{
A 5-bit, 1.6ps resolution true time delay optical beamforming network for 4-element antenna arrays
}

\author{
Laurens Bogaert \\ Ghent University-imec \\ 9052 Ghent, Belgium \\ Laurens.Bogaert@UGent.be
}

Igor Lima de Paula

Ghent University-imec

9052 Ghent, Belgium

Igor.LimadePaula@UGent.be

\author{
Olivier Caytan \\ Ghent University-imec \\ 9052 Ghent, Belgium
}

Olivier.Caytan@UGent.be

\author{
Joris Van Kerrebrouck \\ Ghent University-imec \\ 9052 Ghent, Belgium \\ Joris.VanKerrebrouck@UGent.be
}

\author{
Muhammad Muneeb \\ Ghent University-imec \\ 9052 Ghent, Belgium \\ Muhammad.Muneeb@UGent.be
}

\author{
Quinten Van den Brande \\ Ghent University-imec \\ 9052 Ghent, Belgium \\ Quinten.VandenBrande@UGent.be
}

\author{
Hendrik Rogier \\ Ghent University-imec \\ 9052 Ghent, Belgium \\ Hendrik.Rogier@UGent.be
}

\author{
Guy Torfs \\ Ghent University-imec \\ 9052 Ghent, Belgium \\ Guy.Torfs@UGent.be \\ Johan Bauwelinck \\ Ghent University-imec \\ 9052 Ghent, Belgium \\ Johan.Bauwelinck@UGent.be
}

\author{
Sam Lemey \\ Ghent University-imec \\ 9052 Ghent, Belgium \\ Sam.Lemey@UGent.be
}

\author{
Günther Roelkens \\ Ghent University-imec \\ 9052 Ghent, Belgium \\ Gunther.Roelkens@UGent.be
}

\begin{abstract}
Next-generation mobile networks will use mmWave frequencies as part of their strategy to meet the ever increasing wireless data rate demands. To mitigate the challenging propagation characteristics at these high frequencies, beamforming will be key. In this paper, we discuss a true time delay optical beamforming network (OBFN) based on switchable optical delay lines for a $4 \times 1$ antenna array. This OBFN is implemented on a Si-Photonics platform and the delay lines are capable of discretely tuning the delay difference between elements with a resolution of $1.6 \mathrm{ps}$ up to a maximum delay of $49.6 \mathrm{ps}$.

Index Terms-Analog Radio-over-Fiber (ARoF), Optical Beamforming Network (OBFN), Silicon Photonics, Switchable Optical Delay Lines (SODL), True Time Delay (TTD)
\end{abstract}

\section{INTRODUCTION}

Cellular networks for 5G and beyond tend to keep up with the ever-increasing demand for high-speed wireless data rates by combining several key enabling technologies, such as ultradensification, and the shift towards higher carrier frequencies in the mmWave or even $\mathrm{THz}$ band [1]. To ensure that the small-cell approach is economically viable, Radio-over-Fiber (RoF) schemes have been explored extensively in literature [2]. In such a RoF architecture, the core functionality is centralized and optical communication is used to connect the central office (CO) to the antenna sites. RF-over-Fiber, a version of analog RoF where the RF signal modulated on the optical carrier is already at the correct wireless frequency, is in particular interesting as it omits the need for power-hungry frequency mixers at the Remote Antenna Units (RAUs), thereby enabling low-cost, low-complexity and compact RAUs. In its most basic form, it only requires conversion from optical to electrical domain (and vice versa), amplification of the electrical RF signal

Ghent University (BOF14/GOA/034), ERC Grant ATTO (695495), and Methusalem funding (Grant "Smart Photonic Chips"). and an antenna. The second technique critical for achieving high data rates is the migration towards higher frequencies. This frequency shift offers larger contiguous bandwidths and suffers much less from congestion of the spectrum compared to the traditional sub- $6 \mathrm{GHz}$ bands. Unfortunately, at high carrier frequencies, propagation conditions are much more challenging and introduce increased path losses [3]. On the other hand, the wavelength is much smaller than for traditional sub-6 $\mathrm{GHz}$ antenna systems, which allows for integration of a large number of antenna elements into an array. To mitigate the problem of increased wireless losses, beamforming techniques can be implemented in these antenna arrays [4].

Two main architectural trade-offs are to be made for the beamformer in the context of a mmWave-over-Fiber link [5]. First, the location of the beamforming network, since it can be centralized at the $\mathrm{CO}$ or distributed at the RAUs. Second, the optimal implementation domain: optical or electrical. Due to the possibility of having to address large antenna arrays, we have opted to add the beamforming functionality to the RAU. The result is that only one RF signal per user needs to be transmitted over the RoF link, while centralized beamforming would require sending one RF signal per antenna element over the RoF link, resulting in a significant increase in data to be transferred between CO and RAU. Regarding the preferred domain, optical beamforming networks (OBFN) are likely to outperform electrical variants on several fronts: lower insertion loss, higher bandwidth, reduced size, weight, and power consumption (SWaP), immunity to electromagnetic interference, etc [6]. Furthermore, as carrier frequencies are likely to increase for future generation mobile networks, the high processing bandwidth of microwave photonics seems to provide a future-proof implementation strategy. 


\section{TTD OBFN: DESIGN AND FABRICATION}

In the introduction, it was established that the beamforming functionality in a mmWave-over-Fiber link is best implemented optically at the RAU. Consequently, the downlink RF signal (Fig. 1) is generated at the CO, modulated on an optical carrier, and transported over fiber. When it arrives at the RAU, the signal is split (equally) over the antenna elements and the beamforming coefficients are applied. Finally, at every antenna element, the signal is converted to the electrical domain and passed on to the wireless channel. An elegant way to apply the beamforming coefficients is by introducing reconfigurable optical delay lines between the outputs of the power splitting tree and the antenna elements. The technique of adjusting the delay for every element is called true time delay (TTD) and offers a broadband beamforming approach without beam squint [7]. The steering angle $\theta$ depends on the delay difference between consecutive antenna elements $\Delta T$ and the antenna spacing $d$ as described in eq. 1 [7].

$$
\sin (\theta)=\frac{c \Delta T}{d}
$$

Two variants of TTD are predominantly used in OBFNs. First, switchable optical delay lines (SODLs) [8], [9] offer a straightforward implementation to digitally tune the optical delay by setting switch states to select shorter or longer optical path lengths (Fig. 2). This strategy allows for discrete delay tuning with a tuning resolution $\Delta \mathrm{T}$ and a tuning range of $\left(2^{N}-1\right) \times \Delta \mathrm{T}$, with $\mathrm{N}$ the total number of bits. In a TTD OBFN, the steering angle $\theta$ will be determined by the delay difference between the elements and not by the absolute delay (eq. 1), hence the offset delay $T_{0}$ is irrelevant as long as it is the same for every antenna element.

A second technique to construct optical TTD circuits relies on

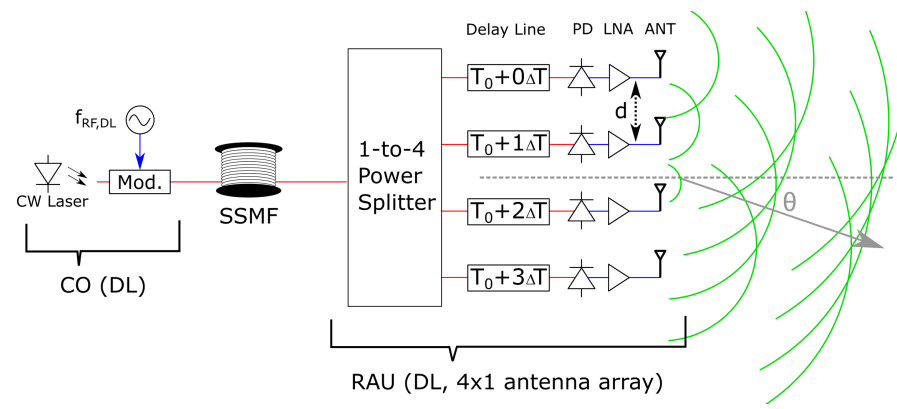

Fig. 1: Downlink mmWave-over-Fiber - CW: Continuous Wave; Mod: Modulator; SSMF: Standard Single Mode Fiber; PD: Photodiode; LNA: Low Noise Amplifier; ANT: Antenna.

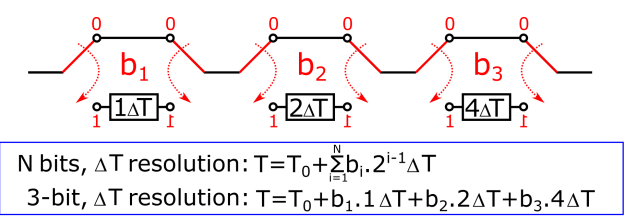

Fig. 2: Operation principle 3-bit standard SODL.
Optical Ring Resonators (ORRs) [10]. By tuning the coupling between a microring and its bus waveguide, one can tune the Q-factor of the ring and consequently continuously tune the group delay for the light to pass the ORR. In this work, we implemented the SODL strategy because the ORR technique results in optically narrowband delay cells.

The SODL in this work was implemented in a silicon photonics platform as its high index contrast enables small bend radii with low loss resulting in compact, low-loss delay lines. The switches are implemented as thermally tunable MachZehnder interferometers (MZIs) and are therefore compact and introduce low-loss switching, with switching speeds on the order of $10 \mu \mathrm{s}$. In the proposed OBFN, an alternative version of the SODL was used (Fig. 3). In the standard SODL, 2 switches are required per bit. A switch is required at the input of every bit, to select between the short and long optical path for that specific bit, and at the output of every bit, to recombine the two paths without excessive loss. The alternative architecture (Fig. 3) only requires a single two-input, two-output switch per bit and as a result the required number of control voltages is reduced. Unfortunately, with this new architecture, the previous bits will determine at what input port of the switch the light will be present and therefore the switch setting ('bar' or 'cross' state) will not only be determined by the required delay for the current bit, but also by all the previous bits. At the output we used a 3-dB combiner instead of a final switch. This design choice was taken to minimize the number of control signals needed to correctly set the delay setting, but will be replaced by an additional switch in future iterations of the design to avoid the inherent $3-\mathrm{dB}$ insertion loss. The adopted SODL design also includes directional couplers tapping off $2.5 \%$ of the light in the waveguide. These power taps make it possible to perform post-fabrication calibration of the switch states.

The proposed OBFN is implemented on imec's passive silicon photonics platform that offers a silicon on insulator (SOI) stack, where the functional Si layer has a thickness of 220nm and allows for both rib and strip waveguides. In the demonstrated OBFN, we used the standard C-band strip waveguides with a height of $220 \mathrm{~nm}$ and a width of $450 \mathrm{~nm}$. These waveguides have a loss of $1.3 \mathrm{~dB} / \mathrm{cm}$, a group index of 4.26 at $1550 \mathrm{~nm}$ and bend losses of only $0.009 \mathrm{~dB}$ per 90 degree bend given a $5 \mu \mathrm{m}$ bend radius. Based on the group index, this $\mathrm{C}$-band waveguide results in approximately 1 ps delay per $70 \mu \mathrm{m}$. An annotated microscope image of the fabricated passive Si-Photonics chip is shown in Fig. 4. This design is optimized for a $4 \times 1$ linear antenna array and

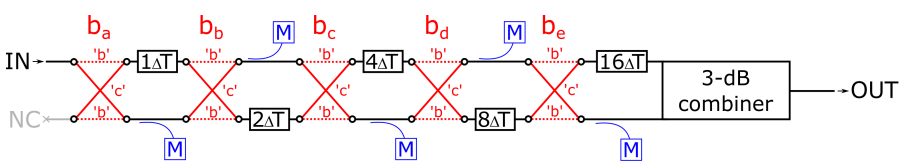

Fig. 3: Adopted 5-bit SODL - M: power monitor port; NC: not connected; 'b': bar state; 'c': cross state. 
contains delay lines with 5-bit discrete tunability and a delay resolution of $1.6 \mathrm{ps}$, resulting in a maximum delay of $49.6 \mathrm{ps}$. The total chip area is $4.7 \mathrm{~mm}^{2}$ and the optical I/O of the OBFN is achieved by using a grating coupler array. Two metal layers were added during post-processing of the SOI chip shown in Fig. 4. First a heater layer $(150 \mathrm{~nm} \mathrm{Ti}+15 \mathrm{~nm} \mathrm{Au})$ was added to enable thermal tuning of the MZI, second, metal routing and bondpads were added in an additional layer $(40 \mathrm{~nm} \mathrm{Ti} \mathrm{+}$ $800 \mathrm{~nm} \mathrm{Au}$ ). The deposited heaters are approximately $500 \mu \mathrm{m}$ long and $2 \mu \mathrm{m}$ wide and result in resistances of about $1050 \Omega$. Figure 5 shows a microscope image of the circuit for a single bit, after metallization.

\section{TTD OBFN: CHARACTERIZATION}

The proposed OBFN in Fig. 4 consists of 4 SODLs. Switch voltages were determined before attaching the fiber array as this attachment covers the monitor ports. In future versions of the OBFN, replacing the monitor ports with photodiodes will help to provide real time feedback of the required bar and cross switch voltages. This makes the design more robust to temperature and wavelength variations. During a calibration measurement, we determined the required voltages at room temperature $\left(25^{\circ} \mathrm{C}\right)$ and for a wavelength of $1550 \mathrm{~nm}$. Based on the extracted switch voltages, we found that the maximum required voltage level is $5.9 \mathrm{~V}$ and that on average, $82.5 \mathrm{~mW}$ per channel is required. This value is equivalent to about $16.5 \mathrm{~mW}$ per switch and in total 20 control voltages are needed to set the 4-channel OBFN.

The operation of the delay line was verified using the setup in Fig. 6. A $28 \mathrm{GHz}$ sine was modulated on a $1550 \mathrm{~nm}$ optical carrier. A fraction of the power (10\%) was tapped off and immediately converted back to the electrical domain, while the main part of the light (90\%) passed the SODL set to the relevant delay setting. Subsequently, this delay path was also converted to the electrical domain and the delay difference between delayed and reference path was measured in a real time oscilloscope, and this was repeated for every delay setting. In Fig. 7, the measured and expected delays are compared to each other. In this plot, the short delay path is

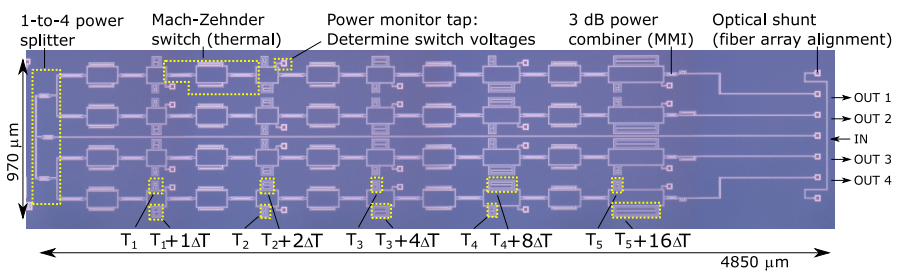

Fig. 4: Layout view and microscope image of the passive SiPhotonics chip before metal deposition.

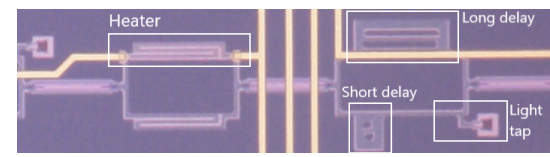

Fig. 5: Microscope image of 1 bit of the SODL after metal deposition: switch to select long or short optical waveguide. used to determine the $T_{0}$ offset and this is used to compensate the 31 other delay settings. Furthermore, the setup described above only provides delay modulo the period of the adopted sine, and therefore, we added a sine period where necessary (i.e. at delay settings beyond $35.7 \mathrm{ps}$ ).

Insertion loss of the OBFN consists of 3 main contributors. First, the chip coupling, which is now performed using grating couplers and results in a total fiber-to-fiber loss of $12 \mathrm{~dB}$ at $1550 \mathrm{~nm}$. This contribution can be greatly reduced by using edge couplers $(<2 \mathrm{~dB}$ per coupler). Second, there is an inherent splitting loss ( $6 \mathrm{~dB}$ for 4 antenna elements). Third, the losses in the SODL should be considered. These are caused by losses in the switches $(0.5 \mathrm{~dB}$ per switch), waveguide losses $(1.3 \mathrm{~dB}$ per $\mathrm{cm})$, bend losses $(0.009 \mathrm{~dB}$ per 90 degree bend), and the inherent $3 \mathrm{~dB}$ loss in the output power combiner. Longer delay sections introduce larger waveguide losses. On the other hand, the introduction of power taps in the short delay path introduces an excess $0.11 \mathrm{~dB}$ loss per tap. Overall, an insertion loss between 7.225 and $7.59 \mathrm{~dB}$ can be observed for the proposed SODL, meaning that loss variations between different delay settings are limited to only $0.365 \mathrm{~dB}$. The insertion loss can be reduced by about $2.5 \mathrm{~dB}$ when replacing the output $3 \mathrm{~dB}$ combiner by an additional switch. This comes at the cost of an additional control voltage per channel and an increased power consumption of about $16.5 \mathrm{~mW}$ per channel.

\section{BeAmsteering Demonstration}

Finally, an OBFN assembly was constructed by bonding the photonic chip to a $\mathrm{PCB}$, attaching a fiber array to the I/O grating couplers, wirebonding the switch bondpads to the PCB and soldering a pin header to easily access the switch control pins. This assembly was subsequently utilized in a mmWave-over-Fiber link similar to Fig. 1. The adopted modulator was a quadrature-biased MZM, an EDFA was used in front of the OBFN (output power set to $16 \mathrm{dBm}$ ) to partially compensate for OBFN losses, and finally free space

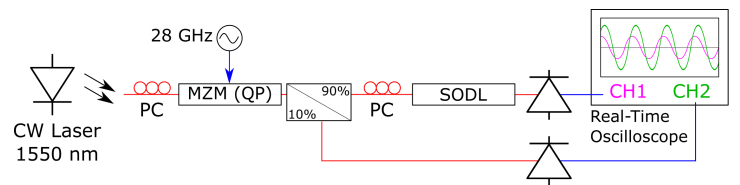

Fig. 6: Setup to measure the delay - MZM: Mach-Zehnder Modulator; QP: Quadrature Point; PC: Polarization Controller

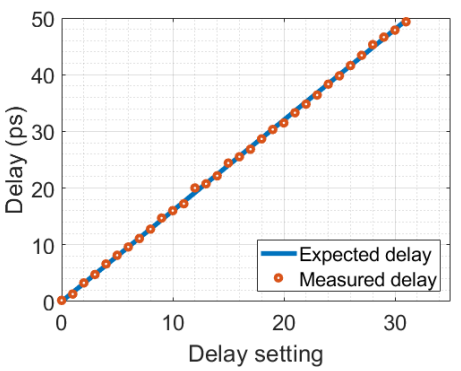

Fig. 7: SODL: comparison of measured and expected delay 


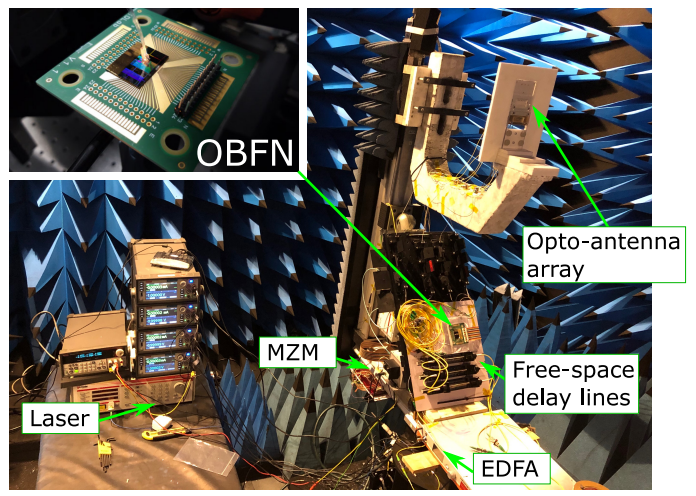

Fig. 8: Anechoic setup for radiation pattern measurements

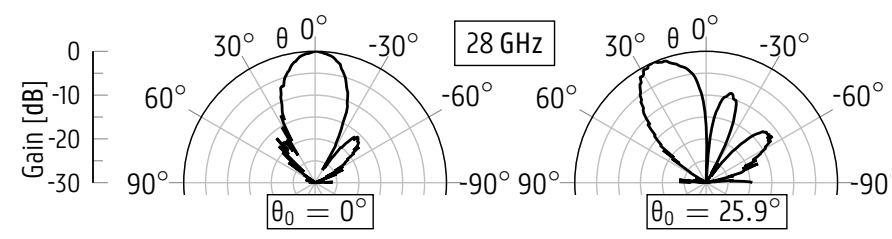

Fig. 9: Normalised measured radiation patterns at $28 \mathrm{GHz}$.

tunable delay lines were introduced between the OBFN and photodiodes to approximately compensate for differences in fiber lengths in the setup. Figure 8 shows the setup that was used to measure the radiation patterns in an anechoic chamber. For this experiment, a $4 \times 1$ opto-antenna array with an antenna spacing of $5.5 \mathrm{~mm}$ was used. The opto-antenna comprises the PD, LNA and ANT functionality depicted in Fig. 1 and therefore contains all elements that are required in a RAU without beamforming. Two radiation patterns obtained with this setup are shown in Fig. 9. First, all SODLs were set to minimal path length, by configuring all switches to their cross state. The signals should then arrive in the optoantenna without relative delay difference between the antenna elements. This is in practice not the case due to variations in fiber length between the OBFN and the PDs. Hence, the free space delay lines were calibrated in this setting to achieve maximal transmission in the broadside direction of the antenna array $\left(\theta=0^{\circ}\right)$. The resulting radiation pattern is shown in Fig. 9 , and from this point on, the free space delay lines were kept fixed. Next, the SODLs in the OBFN were reconfigured by applying the correct switch voltages to achieve $\Delta \mathrm{T}=8 \mathrm{ps}$ (i.e. $0 \mathrm{ps}, 8 \mathrm{ps}, 16 \mathrm{ps}$, and $24 \mathrm{ps}$ for the 4 delay lines). Due to the antenna spacing of $5.5 \mathrm{~mm}$, this corresponds with a steering angle of $25.87^{\circ}$. The resulting radiation pattern is also shown in Fig. 9, and it is clear that the beam is steered towards an angle $\theta$ of approximately $26^{\circ}$. This result shows that the SODL based TTD architecture can perform the beamforming functionality required for mmWave-over-Fiber links.

\section{CONCLUSION}

We have shown that switchable optical delay lines with binary weighted delay settings and thermally steered MachZehnder switches can provide a straightforward implemen- tation of the true time delay circuit that can be used for squint-free broadband beamforming. The proposed OBFN is designed to perform beamsteering of a $4 \times 1$ linear antenna array by changing the relative delay between antenna elements. Discrete 5-bit delay tuning with a resolution of $1.6 \mathrm{ps}$ and a tuning range of $49.6 \mathrm{ps}$ is demonstrated with the fabricated delay lines and furthermore it was shown that beamsteering is possible with this OBFN.

A first improvement to this chip in future developments consists of reducing the insertion loss by replacing the output 3-dB combiner with an additional switch and adopting edge couplers instead of grating couplers. Second, monitor photodiodes should be included in future implementations to determine the switch voltages in real time and, consequently, make the circuit more robust to changes in temperature and wavelength. Adding these monitor photodiodes can be done by migrating the design to imec's full Si-Photonics platform iSiPP50G that includes passive and active components, or by making use of transfer printing to print the photodiodes on top of the grating couplers currently used for calibration of the circuit [11]. Third, transfer printing of semiconductor optical amplifiers (SOAs) at each channel output can help to compensate for the inherent input splitting loss of the OBFN [12]. This will become essential for antenna arrays with a large number of elements.

\section{REFERENCES}

[1] J. G. Andrews, S. Buzzi, W. Choi, S. V. Hanly, A. Lozano, et al., "What Will 5G Be?," IEEE J. Sel. Areas Commun., vol. 32, pp. 1065-1082, June 2014.

[2] V. A. Thomas, M. El-Hajjar, and L. Hanzo, "Performance Improvement and Cost Reduction Techniques for Radio Over Fiber Communications?," IEEE Commun. Surv. Tutor., vol. 17, pp. 627-670, 2015.

[3] S. Rangan, T. S. Rappaport, and E. Erkip, "Millimeter-Wave Cellular Wireless Networks: Potentials and Challenges," Proc. IEEE, vol. 102, pp. 366-385, Mar. 2014.

[4] R. W. Heath Jr., N. Gonzalez-Prelcic, S. Rangan, W. Roh, and A. M. Sayeed, "An Overview of Signal Processing Techniques for Millimeter Wave MIMO Systems," IEEE J. Sel. Topics Signal Process., vol. 10, pp. 436-453, Apr. 2016.

[5] L. Bogaert, J. V. Kerrebrouck, H. Li, I. L. de Paula, K. V. Gasse, et al., "SiPhotonics/GaAs 28-GHz Transceiver With Reflective EAM for Laser-Less mmWave-Over-Fiber," J. Light. Technol., vol. 39, pp. 779786, Feb. 2021.

[6] R. A. Minasian, "Photonic Signal Processing of Microwave Signals," IEEE Trans. Microw. Theory Techn., vol. 54, pp. 832-846, Feb. 2006.

[7] M. Longbrake, "True Time-Delay Beamsteering for Radar," IEEE NAECON, Jul. 2012.

[8] P. Zheng, C. Wang, X. Xu, J. Li, D. Lin, et al., "A Seven Bit Silicon Optical True Time Delay Line for Ka-Band Phased Array Antenna," IEEE Photonics J., vol. 11, Aug. 2019.

[9] C. Zhu, L. Lu, W. Shan, W. Xu, G. Zhou, et al., "Silicon integrated microwave photonic beamformer," Optica, vol. 7, pp. 1162-1170, Sep. 2020.

[10] G. Choo, C. K. Madsen, S. Palermo, and K. Entesari, "Automatic Monitor-Based Tuning of an RF Silicon Photonic $1 \times 4$ Asymmetric Binary Tree True-Time-Delay Beamforming Network," J. Light. Technol., vol. 36, pp. 5263-5275, Nov. 2018.

[11] G. Muliuk, K. V. Gasse, J. V. Kerrebrouck, A. J. Trindade, B. Corbett, et al., " $4 \times 25$ Gbps Polarization Diversity Silicon Photonics Receiver With Transfer Printed III-V Photodiodes," IEEE Photon. Technol. Lett., vol. 31, pp. 287-290, Feb. 2019.

[12] B. Haq, S. Kumari, K. V. Gasse, J. Zhang, A. Gocalinska, et al., "MicroTransfer-Printed III-V-on-Silicon C-Band Semiconductor Optical Amplifiers," Laser Photonics Rev., vol. 14, July 2020. 